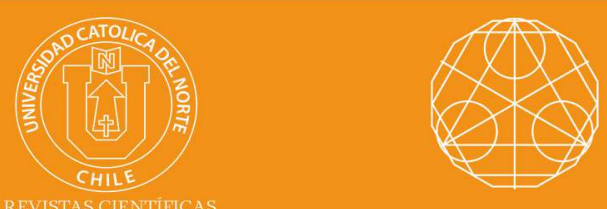

\title{
Extended results on sum divisor cordial labeling
}

\section{A. Sugumaran* (iD orcid.org/0000-0003-3382-4439 \\ K. Rajesh $^{* *} \quad$ (iD) orcid.org/0000-0002-5586-5699}

*Government Arts College, Dept. of Mathematics.Tiruvannamalai, TN, India.

$\checkmark$ sugumaranaruna@gmail.com

**Government Arts College, Dept. of Mathematics.Tiruvannamalai, TN, India.

M.rajesh3429@gmail.com

\section{Received: April 2018 | Accepted: July 2019}

\section{Abstract:}

$A$ sum divisor cordial labeling of a graph $G$ with vertex set $V(G)$ is a bijection $f: V(G) \rightarrow\{1,2, \ldots,|V(G)|\}$ such that an edge uv assigned the label 1 if 2 divides $f(u)+f(v)$ and 0 otherwise. Further the number of edges labeled with 0 and the the number of edges labeled with 1 differ by atmost 1. A graph with sum divisor cordial labeling is called a sum divisor cordial graph. In this paper we prove that the graphs $P_{n}+P_{n}$ ( $n$ is odd), $P_{n} @ K_{1, m}, C_{n} @ K_{1, m}$ ( $n$ is odd), $W_{n} * K_{1, m}$ ( $n$ is even), $<K_{1, n, n}^{1} \triangle K_{1, n, n}^{2}>,<F l_{n}^{1} \triangle F l_{n}^{2}>$ are sum divisor cordial graphs.

Keywords: Divisor cordial labeling; Sum divisor cordial labeling.

$\operatorname{MSC}(2010): 05 C 78$.

\section{Cite this article as (IEEE citation style):}

A. Sugumaran and K. Rajesh," Extended results on sum divisor cordial labeling", Proyecciones (Antofagasta, On line), vol. 38 , no. 4, pp. 653-663, Oct. 2019, doi: 10.22199/issn.07176279-2019-04-0042. [Accessed dd-mm-yyyy]

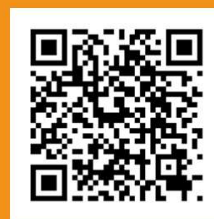

Article copyright: (c) 2019 Aruna Sugumaran and Karunanithi Rajesh. This is an open access article distributed under the terms of the Creative Commons Licence, which permits unrestricted use and distribution provided the original author and source are credited. 


\section{Introduction}

By a graph, we mean a finite undirected graph without loops or multiple edges. For standard terminology and notations related to graph theory, we refer to Harary[2]. A labeling of graph is a map that carries the graph elements to the set of numbers, usually to the set of non-negative or positive integers. If the domain is the set of edges, then we speak about edge labeling. If the labels are assigned to both vertices and edges, then the labeling is called total labeling. Cordial labeling is further extended to divisor cordial labeling, prime cordial labeling, total cordial labeling, Fibonacci cordial labeling etc.,

Varatharajan et al. [11] introduced the concept of divisor cordial labeling. For dynamic survey of various graph labeling, we refer to Gallian [1]. Lourdusamy and Patrick [5] introduced the concept of sum divisor cordial labeling. Sugumaran and Rajesh [6] have proved that Swastik graph $S w_{n}$, path union of finite copies Swastik graph $S w_{n}$, cycle of $k$ copies of Swastik graph $S w_{n}$ ( $k$ is odd), Jelly fish $J(n, n)$ and Petersen graph are sum divisor cordial graphs. Sugumaran and Rajesh [7] have proved that the Theta graph and some of the graph operations of Theta graph are sum divisor cordial graphs. Sugumaran and Rajesh [8] have proved that the Herschel graph and some of the graph operations of Herschel graph are sum divisor cordial graphs. Sugumaran and Rajesh [9] have proved that $H_{n}$ ( $n$ is odd), $C_{3} @ K_{1, n},<F_{n}^{1} \triangle F_{n}^{2}>$, open star of Swastik graph $S\left(t . S w_{n}\right)$, when $t$ is odd are sum divisor cordial graphs. Sugumaran and Rajesh [10] have proved that some graph operations related to $H$-graph are sum divisor cordial graphs. In this paper we investigate sum divisor cordial labeling of the graphs such as $P_{n}+P_{n}$ ( $n$ is odd), $P_{n} @ K_{1, m}, C_{n} @ K_{1, m}$ ( $n$ is odd), $W_{n} * K_{1, m}$ ( $n$ is even), $<K_{1, n, n}^{1} \triangle K_{1, n, n}^{2}>$ and $<F l_{n}^{1} \triangle F l_{n}^{2}>$.

Definition 1.1. If the vertices are assigned values subject to certain conditions then it is known as graph labeling.

Definition 1.2. A mapping $f: V(G) \rightarrow\{0,1\}$ is called binary vertex labeling of $G$ and $f(v)$ is called the label of the vertex $v$ of $G$ under $f$.

Notation. If for an edge $e=u v$, the induced edge labeling $f^{*}: E(G) \rightarrow$ $\{0,1\}$ is given by $f^{*}(e)=|f(u)-f(v)|$, then we denote $v_{f}(i)=$ number of vertices of $G$ having label $i$ under $f$, 
$e_{f}(i)=$ number of vertices of $G$ having label $i$ under $f^{*}$.

Definition 1.3. A binary vertex labeling $f$ of a graph $G$ is called a cordial labeling if $\left|v_{f}(0)-v_{f}(1)\right| \leq 1$ and $\left|e_{f}(0)-e_{f}(1)\right| \leq 1$. A graph $G$ is cordial if it admits a cordial labeling.

Definition 1.4. [11] Let $G=(V(G), E(G))$ be a simple graph and $f$ : $V \rightarrow\{1,2, \ldots,|V(G)|\}$ be a bijection. For each edge uv, assign the label 1 if either $f(u) \mid f(v)$ or $f(v) \mid f(u)$ and the label 0 otherwise. The function $f$ is called a divisor cordial labeling if $\left|e_{f}(0)-e_{f}(1)\right| \leq 1$. A graph which admits a divisor cordial labeling is called a divisor cordial graph.

Definition 1.5. Let $G=(V(G), E(G))$ be a simple graph and let $f: V \rightarrow$ $\{1,2, \ldots,|V(G)|\}$ be a bijection. For each edge uv, assign the label 1 if $2 \mid(f(u)+f(v))$ and the label 0 otherwise. The function $f$ is called a sum divisor cordial labeling if $\left|e_{f}(0)-e_{f}(1)\right| \leq 1$. A graph which admits a sum divisor cordial labeling is called a sum divisor cordial graph.

Definition 1.6. Let $G$ be a simple and connected graph. The graph $G @ K_{1, n}$ is obtained from attaching the apex vertex of a copy of $K_{1, n}$ at each vertex of $G$.

Definition 1.7. $W_{n} * K_{1, m}$ is the graph obtained from the wheel $W_{n}$ by attaching the apex vertex of a copy of $K_{1, m}$ at each rim vertex of $W_{n}$.

Definition 1.8. Let $G$ be a simple and connected graph. Consider two copies of graph $G$ namely $G_{1}$ and $G_{2}$. Then the graph $G^{\prime}=<G_{1} \triangle G_{2}>$ is the graph obtained by joining the apex/central vertices of $G_{1}$ and $G_{2}$ by an edge as well as to a new vertex $x$.

\section{Main Results}

Theorem 2.1. The connected graph $P_{n}+P_{n}$ is a sum divisor cordial labeling, where $n$ is odd.

Proof. Let $G=P_{n}+P_{n}$ be a connected graph with vertex set $\left\{v_{1}, v_{2}, \ldots, v_{n}, v_{n+1}, \ldots, v_{2 n}\right\}$. Then $G$ has $2 n$ vertices and $n^{2}+2 n-2$ edges. We define the vertex labeling $f: V(G) \rightarrow\{1,2, \ldots,|V(G)|\}$ as follows: $f\left(v_{i}\right)=i, i \equiv 1(\bmod 4)$. 
$f\left(v_{i}\right)=i, i \equiv 2(\bmod 4)$.

$f\left(v_{i}\right)=i+1, i \equiv 3(\bmod 4)$.

$f\left(v_{i}\right)=i-1, i \equiv 0(\bmod 4)$.

From the above labeling pattern, we have $\left|e_{f}(0)-e_{f}(1)\right| \leq 1$.

Hence $G$ is a sum divisor cordial graph.

Example 2.2. The sum divisor cordial labeling of bistar $P_{5}+P_{5}$ is shown in Figure 2.1

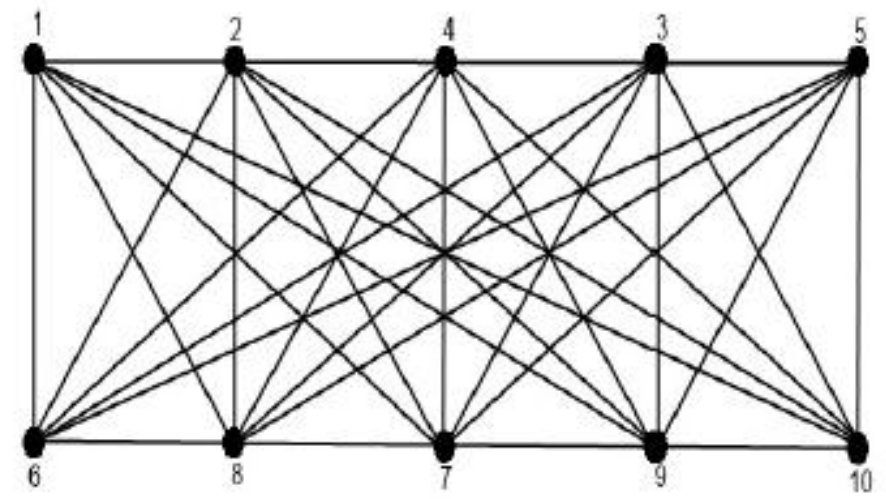

Figure 2.1

Theorem 2.3. The graph $P_{n} @ K_{1, m}$ is a sum divisor cordial labeling.

Proof. Let $G=P_{n} @ K_{1, m}$ and let $u_{1}, u_{2}, \ldots, u_{n}$ be the vertices of $P_{n}$. Let $V(G)=\left\{v, u_{i}, v_{j}^{i}: 1 \leq i \leq n ; 1 \leq j \leq m\right\}$ where $v$ is the apex vertex and $v_{j}^{i}$ is the $j^{\text {th }}$ pendant vertex of the $i^{\text {th }}$ copy of $K_{1, m}(1 \leq i \leq n ; 1 \leq j \leq m)$. In $G, v=u_{i}$. Then $G$ has $n(m+1)$ vertices and $n(m+1)-1$ edges.

We define the vertex labeling $f: V(G) \rightarrow\{1,2, \ldots,|V(G)|\}$ as follows:

Case 1: $n$ is even

$f\left(u_{i}\right)=i, i \equiv 1(\bmod 4)$.

$f\left(u_{i}\right)=i, i \equiv 2(\bmod 4)$.

$f\left(u_{i}\right)=i+1, i \equiv 3(\bmod 4)$.

$f\left(u_{i}\right)=i-1, i \equiv 0(\bmod 4)$.

$f\left(v_{j}^{i}\right)=m(i-1)+n+j, 1 \leq i \leq n ; 1 \leq j \leq m$.

Case 2: $n$ is odd

$f\left(u_{i}\right)=i, i \equiv 1(\bmod 4)$. 
$f\left(u_{i}\right)=i+1, i \equiv 2(\bmod 4)$.

$f\left(u_{i}\right)=i-1, i \equiv 3(\bmod 4)$.

$f\left(u_{i}\right)=i, i \equiv 0(\bmod 4)$.

$f\left(v_{j}^{i}\right)=m(i-1)+n+j, 1 \leq i \leq n ; 1 \leq j \leq m$.

From the above labeling pattern, we have $\left|e_{f}(0)-e_{f}(1)\right| \leq 1$.

Hence $G$ is a sum divisor cordial graph.

Example 2.4. The sum divisor cordial labeling of bistar $P_{4} @ K_{1,5}$ is shown in Figure 2.2

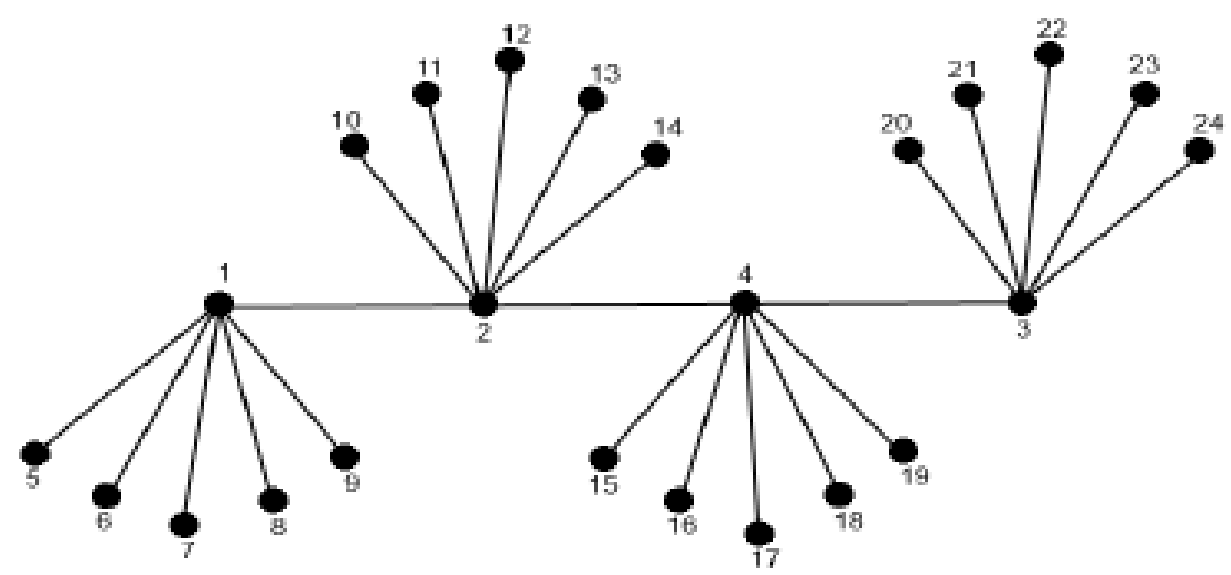

Figure 2.2

Theorem 2.5. The graph $C_{n} @ K_{1, m}$ is a sum divisor cordial labeling, where $n$ is odd and $m$ is any positive integer.

Proof. Let $G=C_{n} @ K_{1, m}$. We denote $v_{j}^{i}$ is the $j^{t h}$ pendant vertex of the $i^{\text {th }}$ copy of $K_{1, m}(1 \leq i \leq n ; 1 \leq j \leq m)$. Let $V(G)=\left\{u, u_{i}, v_{j}^{i}: 1 \leq\right.$ $i \leq n ; 1 \leq j \leq m\}$ where $v$ is the apex vertex of $K_{1, m}$ and in $G, v=u_{i}$ $(1 \leq i \leq n)$. Then $G$ has $n(m+1)$ vertices and $n(m+1)$ edges.

We define the vertex labeling $f: V(G) \rightarrow\{1,2, \ldots,|V(G)|\}$ as follows:

$f\left(u_{i}\right)=i, i \equiv 1(\bmod 4)$.

$f\left(u_{i}\right)=i+1, i \equiv 2(\bmod 4)$.

$f\left(u_{i}\right)=i-1, i \equiv 3(\bmod 4)$.

$f\left(u_{i}\right)=i, i \equiv 0(\bmod 4)$.

$f\left(v_{j}^{i}\right)=m(i-1)+n+j, 1 \leq i \leq n ; 1 \leq j \leq m$. 
From the above labeling pattern, we have $\left|e_{f}(0)-e_{f}(1)\right| \leq 1$.

Hence $G$ is a sum divisor cordial graph.

Example 2.6. The sum divisor cordial labeling of $C_{5} @ K_{1,6}$ is shown in Figure 2.3

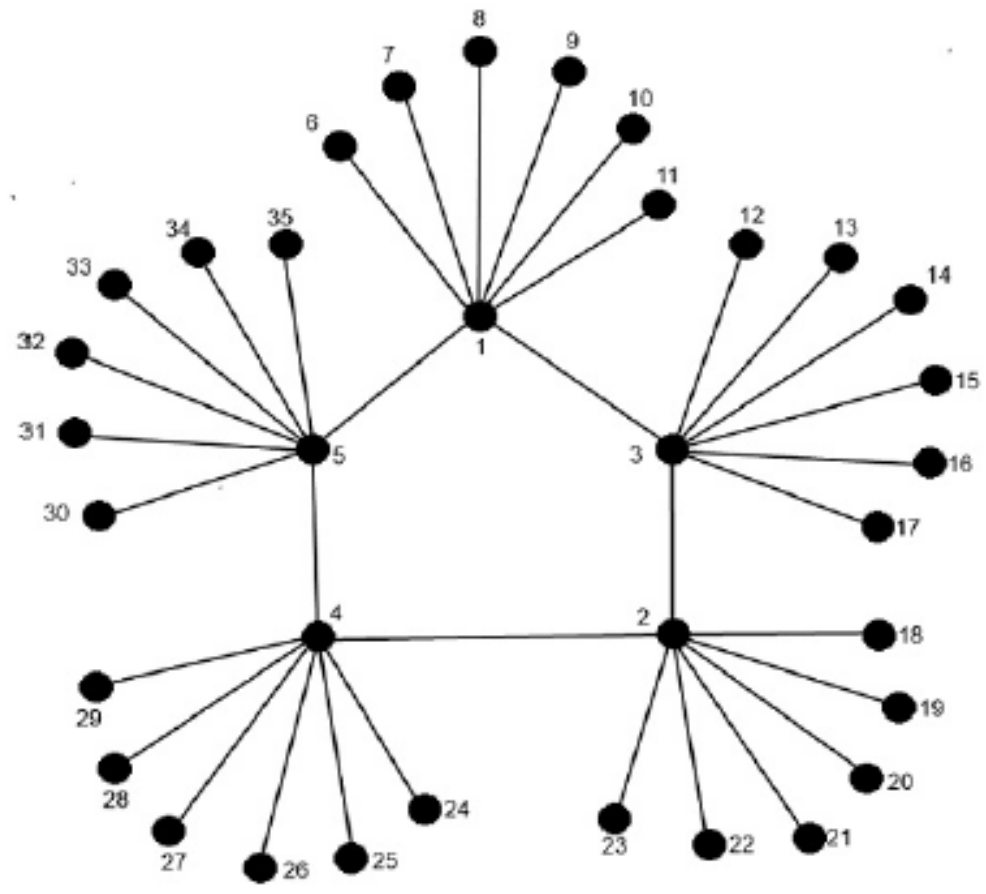

Figure 2.3

Theorem 2.7. The graph $W_{n} * K_{1, m}$ is a sum divisor cordial labeling, where $n$ is even and $m$ is any positive integer.

Proof. Consider $G=W_{n} * K_{1, m}$. We denote $v_{j}^{i}$ is the $j^{\text {th }}$ pendant vertex of the $i^{\text {th }}$ copy of $K_{1, m}$. Let $V(G)=\left\{u, v, u_{i}, v_{j}^{i}: 1 \leq i \leq n ; 1 \leq j \leq m\right\}$ where $u, u_{i}$ are the apex and rim vertices of $W_{n}$ and $v$ is the apex vertex of $K_{1, m}$. In $G, v=u_{i}$ for all $i=1,2, \ldots, n$.

Then $G$ has $n(m+1)+1$ vertices and $n(m+2)$ edges.

We define the vertex labeling $f: V(G) \rightarrow\{1,2, \ldots,|V(G)|\}$ as follows: 
Case 1: When $n \equiv 0(\bmod 4)$

$f(u)=1$,

$f\left(u_{i}\right)=i+1, i \equiv 1(\bmod 4)$.

$f\left(u_{i}\right)=i+2, i \equiv 2(\bmod 4)$.

$f\left(u_{i}\right)=i, i \equiv 3(\bmod 4)$.

$f\left(u_{i}\right)=i+1, i \equiv 0(\bmod 4)$.

$f\left(v_{j}^{i}\right)=m(i-1)+n+j+1,1 \leq i \leq n ; 1 \leq j \leq m$.

Case 2: when $n \equiv 2(\bmod 4)$

$f(u)=2$,

$f\left(u_{i}\right)=i, i \equiv 1(\bmod 4)$.

$f\left(u_{i}\right)=i+1, i \equiv 2(\bmod 4)$.

$f\left(u_{i}\right)=i+1, i \equiv 3(\bmod 4)$.

$f\left(u_{i}\right)=i+2, i \equiv 0(\bmod 4)$.

$f\left(v_{j}^{i}\right)=m(i-1)+n+j+1,1 \leq i \leq n ; 1 \leq j \leq m$.

From the above labeling pattern, we have $\left|e_{f}(0)-e_{f}(1)\right| \leq 1$.

Hence $G$ is a sum divisor cordial graph.

Example 2.8. The sum divisor cordial labeling of $W_{6} * K_{1,4}$ is shown in Figure 2.4 


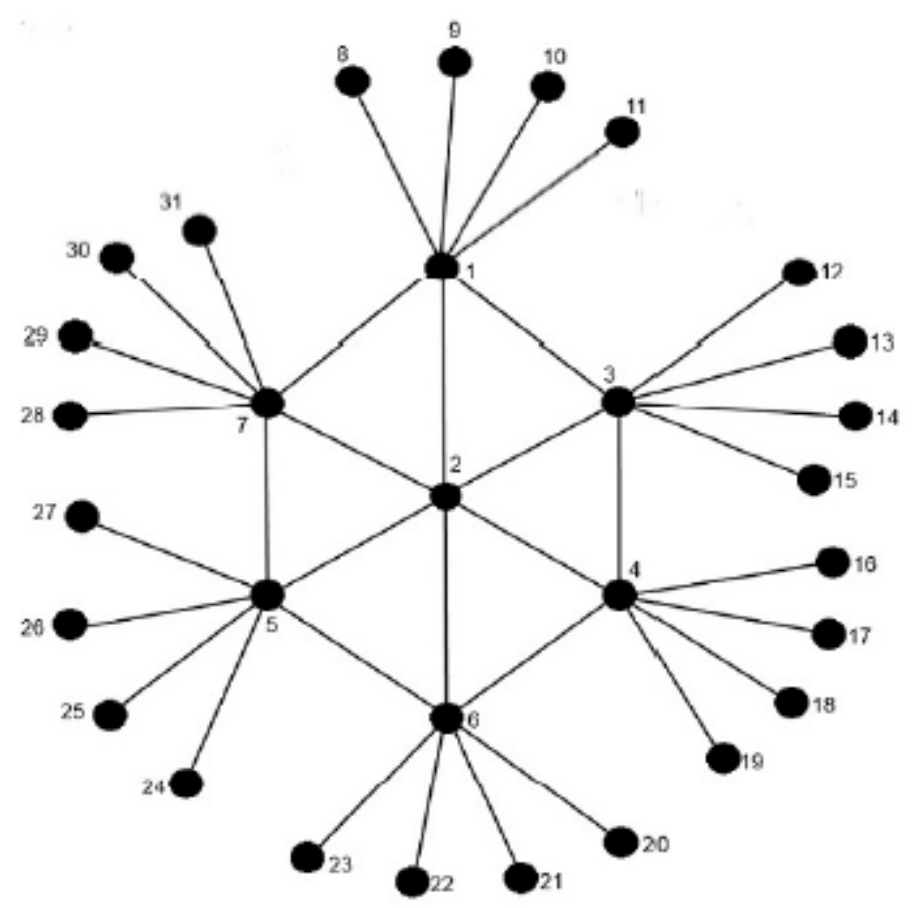

Figure 2.4

Theorem 2.9. The graph $<K_{1, n, n}^{1} \triangle K_{1, n, n}^{2}>$ is a sum divisor cordial labeling.

Proof. $\quad$ Let $G=<K_{1, n, n}^{1} \triangle K_{1, n, n}^{2}>$. Let $V(G)=\left\{x, u, v, u_{i}, v_{i}, u_{i}^{\prime}, v_{i}^{\prime}\right.$ : $1 \leq i$

$\leq n\}$, where $u$ and $v$ are the apex vertices, $u_{i}$ and $v_{i}(1 \leq i \leq n)$ are the first level vertices, $u_{i}^{\prime}$ and $v_{i}^{\prime}(1 \leq i \leq n)$ are the second level vertices in $K_{1, n, n}^{1}$ and $K_{1, n, n}^{2}$ respectively. Also, let $x$ be the new vertex added in $G$. Then $G$ has $4 n+3$ vertices and $4 n+3$ edges.

We define the vertex labeling $f: V(G) \rightarrow\{1,2, \ldots,|V(G)|\}$ as follows:

$f(x)=3$,

$f(u)=2$,

$f(v)=1$,

$f\left(u_{i}\right)=2 i+2,1 \leq i \leq n$.

$f\left(u_{i}^{\prime}\right)=2 i+3,1 \leq i \leq n$.

$f\left(v_{i}\right)=2 n+2 i+3,1 \leq i \leq n$.

$f\left(v_{i}^{\prime}\right)=2 n+2 i+2,1 \leq i \leq n$. 
From the above labeling pattern, we have $\left|e_{f}(0)-e_{f}(1)\right| \leq 1$.

Hence $G$ is a sum divisor cordial graph.

Example 2.10. The sum divisor cordial labeling of $<K_{1,3,3}^{1} \triangle K_{1,3,3}^{2}>$ is shown in Figure 2.5

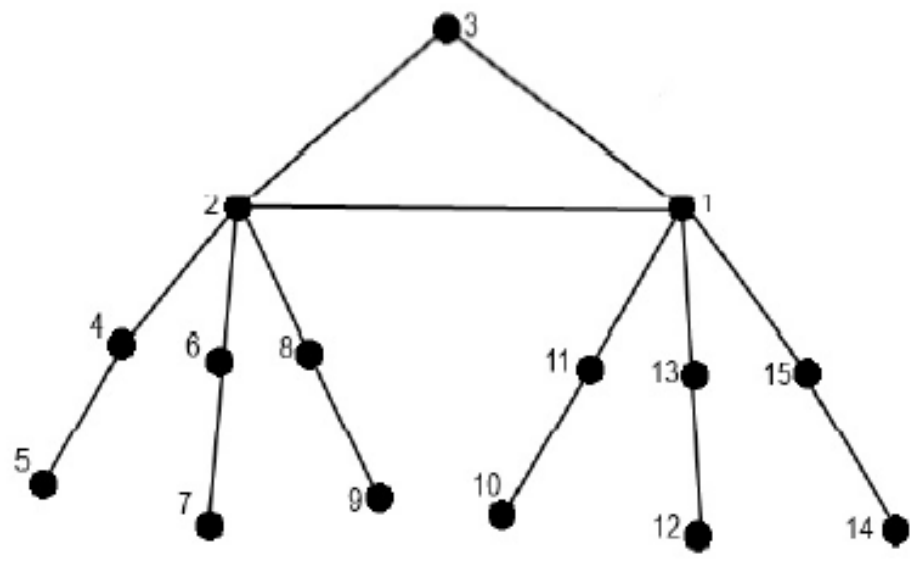

Figure 2.5

Theorem 2.11. The graph $<F l_{n}^{1} \triangle F l_{n}^{2}>$ is a sum divisor cordial labeling.

Proof. Let $G=<F l_{n}^{1} \triangle F l_{n}^{2}>$. Let $V(G)=\left\{x, u, v, u_{i}, v_{i}, u_{i}^{\prime}, v_{i}^{\prime}: 1 \leq\right.$ $i \leq$

$n\}$, where $u$ and $v$ are the central vertices, $u_{i}$ and $v_{i}(1 \leq i \leq n)$ are the rim vertices, $u_{i}^{\prime}$ and $v_{i}^{\prime}(1 \leq i \leq n)$ are the newly added vertices in $F_{n}^{1}$ and $F_{n}^{2}$ respectively. Also, let $x$ be the new vertex added in $G$. Then $G$ has $4 n+3$ vertices and $8 n+3$ edges.

We define the vertex labeling $f: V(G) \rightarrow\{1,2, \ldots,|V(G)|\}$ as follows:

$f(u)=1$,

$f(v)=2 n+3$,

$f(x)=2 n+2$,

$f\left(u_{i}\right)=2 i, 1 \leq i \leq n$.

$f\left(u_{i}^{\prime}\right)=2 i+1,1 \leq i \leq n$.

$f\left(v_{i}\right)=2 n+2 i+2,1 \leq i \leq n$.

$f\left(v_{i}^{\prime}\right)=2 n+2 i+3,1 \leq i \leq n$. 
From the above labeling pattern, we have $\left|e_{f}(0)-e_{f}(1)\right| \leq 1$. Hence $G$ is a sum divisor cordial graph.

Example 2.12. The sum divisor cordial labeling of $<F l_{3}^{1} \triangle F l_{3}^{2}>$ is shown in Figure 2.6

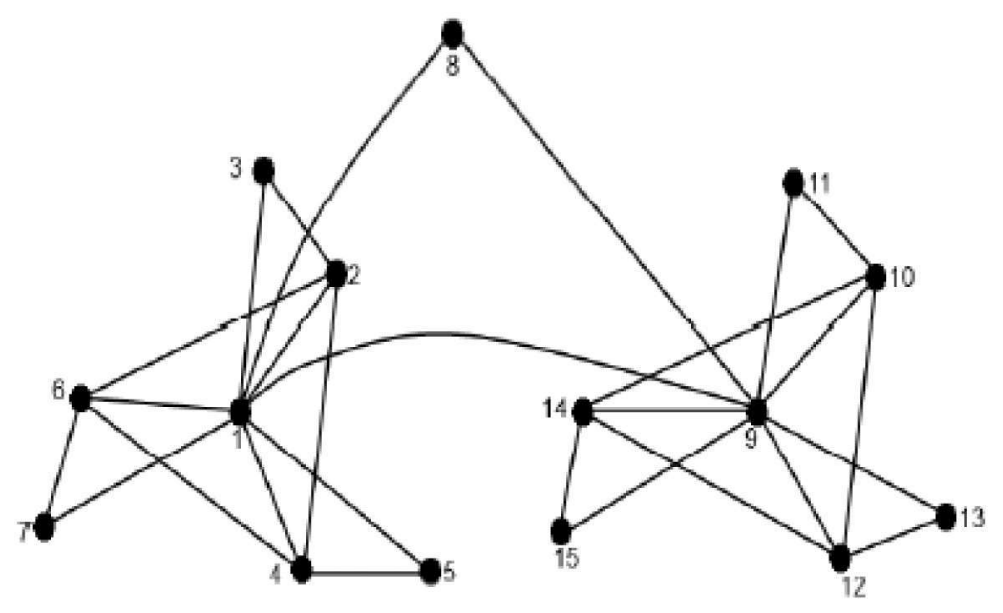

Figure 2.6

\section{Conclusion}

In this paper, we proved that some of the graphs such as $P_{n}+P_{n}$ ( $n$ is odd), $P_{n} @ K_{1, m}, C_{n} @ K_{1, m}$ ( $n$ is odd), $W_{n} * K_{1, m}$ ( $n$ is even), $\left\langle K_{1, n, n}^{1} \triangle K_{1, n, n}^{1}>\right.$, $\left.<F l_{n}^{1} \triangle F l_{n}^{2}\right\rangle$ are sum divisor cordial graphs. It is an interesting open area of research to identify new graphs which admit sum divisor cordial labeling.

\section{References}

[1] J. Gallian, "A Dynamic Survey of Graph Labeling”, 20th ed. The electronics journal of combinatorics, vol. \# DS6, p. 432, 2007. [On line]. Available: http://bit.ly/33bdu1B

[2] F. Harary, Graph theory. Reading, MA: Addison-Wesley, 1972. 
[3] G. Ghodasara and M.. Patel, "Some new combination graphs", International journal of mathematics and its applications, vol. 5, no. 2-A, pp. 153-161, 2017. [On line]. Available: https://bit.ly/2nCU8CS

[4] V. Kaneria and H. Makadia, "Graceful labeling for plus graph", International journal of current research in science and technology, vol.1, no. 3, pp. 15-20, 2015. [On line]. Available: https://bit.ly/2pjtOhu

[5] A. Lourdusamy and F. Patrick, "Sum divisor cordial graphs", Proyecciones (Antofagasta, On line), vol. 35, no. 1, pp. 119-136, Mar. 2017, doi: 10.4067/S0716-09172016000100008.

[6] A. Sugumaran and K. Rajesh,"Some new results on sum divisor cordial graphs", Annals of pure and applied mathematics, vol. 14, no. 1, pp. 45-52, Jul. 2017, doi: 10.22457/apam.v14n1a6.

[7] A. Sugumaran and K. Rajesh, "Sum divisor cordial labeling of Theta graph", Annals of pure and applied mathematics, vol. 14, no. 2, pp. 313-320, Sep. 2017, doi: 10.22457/apam.v14n2a14.

[8] A. Sugumaran and K. Rajesh, "Sum divisor cordial labeling of Herschel graph", Annals of pure and applied mathematics, vol. 14, no.3, pp. 465-472, Oct. 2017, doi: 10.22457/apam.v14n3a14.

[9] A. Sugumaran and K. Rajesh, "Further results on sum divisor cordial labeling", Journal of computer and mathematical sciences, vol. 8, no. 12 , pp. 802-807, Dec. 2017 , doi: $10.29055 / \mathrm{jcms} / 721$.

[10] A. Sugumaran and K. Rajesh, "Some graph operations on sum divisor cordial labeling related to H-graph", International journal of statistics and applied mathematics, vol. 3, no. 1, pp. 389-395, 2018. [On line]. Available: https://bit.ly/2orKq6k

[11] R. Varatharajan, S. Navaneethakrishnan and K. Nagarajan, "Divisor cordial graph", International journal of mathematical combinatorics, vol. 4, pp. 15-25, Dec. 2011. [On line]. Available: https://bit.ly/2pjygwK 